\title{
Treatment Options for Non-Colorectal Cancer Liver Metastases
}

\author{
Xianglong Tan', Mengyang $\mathrm{Li}^{2}$, Zhiming Zhao ${ }^{1 *}$
}

'The Second Department of Hepatopancreatobiliary Surgery, The First Medical Center, Chinese People's Liberation Army General Hospital, Beiiing, China ${ }^{2}$ Department of Hepatopancreatobiliary Surgery, The Fourth Medical Center, Chinese People's Liberation Army General Hospital, Beijing, China

\section{Article Info}

\section{Article Notes}

Received: April 03, 2020

Accepted: April 24, 2020

\section{${ }^{*}$ Correspondence:}

Dr. Zhiming Zhao, The Second Department of Hepatopancreatobiliary Surgery, The First Medical Center, Chinese People's Liberation Army General Hospital, Beijing, China; Email: zhimingzha0616@126.com.

(c) 2020 Zhao Z. This article is distributed under the terms of the Creative Commons Attribution 4.0 International License.

\section{ABSTRACT}

Liver metastases occur during the progression of various malignancies. Similar to colorectal cancer, liver metastases of neuroendocrine cancer, gastrointestinal stromal tumor, and pancreatic cancer also determine patient prognosis to some extent. With the development of surgical techniques, pharmaceutical research, and perioperative treatment, therapeutic strategies for non-colorectal cancer liver metastases have improved greatly during the last two decades, inevitably leading to some controversies. Here, we have reviewed the current treatment options for non-colorectal cancer with the aim of enhancing our understanding of the latest developments in this field.

\section{Introduction}

The liver is one of the most common sites of metastases in various malignancies. Treatment of liver metastases, to some extent, determines the prognosis of patients. Treatment strategies for liver metastatic lesions vary significantly between different tumors, because of their unique biological characteristics. A systematic review of the therapeutic approaches for various cancer-related liver metastases would provide a better understanding of the latest progression and controversies regarding the surgical options, systematic chemotherapy, and palliative treatment. In this review, we have summarized the therapeutic options for non-colorectal cancer liver metastases in neuroendocrine tumors, gastrointestinal stromal tumors, pancreatic cancer, kidney cancer, and breast cancer. In addition, we attempt to answer the following questions for different pathological liver metastases.

1. What are the incidence, prevalence, and prognosis characteristics of different tumor liver metastases?

2. Does surgery improve prognosis?

2. What are the criteria for surgical resection?

4. For each subtype of patients, which is the best surgical approach amongst the available options?

5. What are the perioperative risk factors and complications of different approaches?

6. What is the systematic therapy scheme for different cancer liver metastases?

7. Is there any new prospective approach?

\section{Neuroendocrine tumors (NETs)}

The treatment options for NET liver metastases include surgical 
resection, liver transplantation, loco-regional therapies, and medical treatment.

\section{Surgery}

Curative surgical resection is possible in only $10 \%$ $25 \%$ of NET patients with liver metastases. Saxena et al. summarized 29 studies on NET liver metastases, and reported that $63 \%$ of the patients achieved $\mathrm{R} 0$ resection, with the 5- and 10-year overall survival rates of $70.5 \%$ and $42 \%$, respectively ${ }^{1,2}$. Criteria for patients to receive surgery include: well-differentiated G1/G2 tumors, absence of distant lymph node metastasis, absence of extrahepatic metastasis, absence of diffuse peritoneal metastasis, and absence of right cardiac dysfunction. Although $80 \%$ of the patients experienced recurrence within 5 years after resection, the outcome of liver resection was still better when compared to other treatments ${ }^{3}$. A retrospective analysis of surgery and loco-regional therapy showed that the 5 -year survival rate was $71 \%$ in partial hepatectomy compared to $31 \%$ in liver chemoembolization ${ }^{4,5}$. Debulking surgery, which involves removal of the majority of liver lesions, can release the tumor burden and help in controlling symptoms. Debulking surgery has also been reported to enhance the effect of subsequent chemoembolization ${ }^{4}$. Synchronous resection of the primary tumor and liver metastases showed acceptable complication rates $(18 \%$ $25 \%)$ and favorable 5-year survival rates $(70 \%-71 \%)^{6-10}$. Compared to the non-surgical group, palliative resection of the primary tumor significantly improved the 5-year survival rate $(35.7 \%-83 \%$ versus $5.4 \%-50 \%)$, even in patients with primary tumors and unresectable metastases sites $^{4}$.

Radiofrequency ablation (RFA) can be used in tumors $<5$ $\mathrm{cm}$, which is away from vital structures and liver surface. It is also a good choice for debulking surgery, considering its safety and repeatability ${ }^{11}$. Transarterial chemoembolization (TACE) is another option for palliative therapy since most of the blood supply to the metastatic site comes from the arteries. Doxorubicin, melphalan, and streptozocin are the most commonly used chemotherapeutics for TACE. A recent study showed that the median tumor progression time of TACE treatment was $14 \pm 16$ months and median overall survival was $22 \pm 18$ months ${ }^{12}$.

\section{Liver transplantation}

Except for CRLM, neuroendocrine tumor (NET) liver metastases are brought into indications of liver transplantation. Because of the portal vein drainage of the gastrointestinal tract and pancreas, hematogenous spread of NETs is the most common type of metastasis. Additionally, according to the SEER database, $27 \%$ of NET patients present with liver metastasis at the time of diagnosis. The liver is the most common site for NET metastases (40\%-85\%). Due to their less aggressive biological characteristics, liver transplantation for NETs tends to have better outcomes than for other malignancies.

Mazzaferro et al. developed a fairly strict criteria to select patients with liver metastasis NET for liver transplantation. The inclusion criteria were as follows: age $<55$ years, well-differentiated NET tumor, Ki $67<5 \%$, stable for 6 months before liver transplantation, R0 resection of the primary tumor, less than $50 \%$ liver involvement, and no extrahepatic disease. The exclusion criteria were as follows: noncarcinoid tumor, pre-existing medical condition precluding liver transplantation, and tumor not drained from the portal system. Under the guidance of these criteria, the 5-year overall survival rate was $90 \%$ and the DFS rate was $77 \%{ }^{13}$. Other studies on liver transplantation in NET patients showed that the 5-year overall survival rate in selected patients was $44 \%-52 \%$, which was still much higher than the $20 \%-30 \%$ expected 5 -year survival rate in untreated patients ${ }^{1,14}$. This indicates that liver transplantation may be a good option for unresectable liver metastases of NETs.

\section{Somatostatin analogs}

NETs express at least one of the five somatostatin receptors. Somatostatin analogs can bind to somatostatin receptors, exerting a tumor suppression function. In total, the stabilization rate was $40 \%-80 \%$ and the objective tumor response rate was $10 \%$. A recent randomized control trial reported that in patients who received primary tumor resection with $<10 \%$ liver involvement, somatostatin analogs could significantly reduce the risk of progression $(66.7 \%)^{15-19}$.

\section{Systemic chemotherapy}

Systemic chemotherapy is recommended for advanced and unresectable NETs, especially PNETs. Cytotoxic agents, such as streptozotocin, cisplatin, dacarbazine, doxorubicin, and 5-fluorouracil are the commonly used chemotherapeutic agents ${ }^{19}$. In patients with metastatic, well-differentiated PNETs, treatment with temozolomide in combination with capecitabine led to a partial response rate of $70 \%$, median progression-free survival (PFS) of 18 months, and 2-year survival of $92 \%{ }^{20}$. For patients with high-grade tumors, cisplatin is preferred. The response rate achieved with cisplatin combined with etoposide was $42 \%-67 \%{ }^{21}$.

\section{Targeted therapy}

mTOR, VEGF, and VEGFRupregulation has been observed in PNETs. In the RADIANT-3 clinical trial, treatment with the mTOR inhibitor, everolimus, significantly improved PFS compared to placebo (11 months vs. 4.6 months $)^{4}$. Sunitinib is a multi-tyrosine kinase inhibitor with a high affinity to 
VEGFR. A study demonstrated that the PFS in the sunitinib group was 11.1 months (versus 5.5 months in the placebo group). No evidence exists for the use of everolimus or sunitinib in liver metastases of intestinal origin ${ }^{22}$.

\section{Pancreas}

Unlike the significant improvements achieved in treatment options for other malignancies, the median overall survival in patients with pancreatic ductal carcinoma still remains less than 1 year. The 5 -year overall survival was only $20 \%$, even in patients who underwent radical surgery. In addition, some patients with pancreatic ductal adenocarcinoma (PDAC) were diagnosed with liver metastases. Previously, the median overall survival of patients who received synchronous resection of liver metastases and primary tumor was only 6 months, which is not superior to systematic chemotherapy or palliative bypass $^{23,24}$. Thus, once distant metastases is diagnosed, radical resection is not recommended ${ }^{25}$. However, a recent retrospective study suggested that synchronous or metachronous resection can be performed in selected patients. The median overall survival was 14.5 months in resected patients and 7.5 months in non-surgical patients. The 5-year survival rates were $0 \%$ and $5.8 \%$, respectively. The drawback of this approach was that synchronous resection had a higher morbidity compared to metachronous resection: $33 \%-45 \%$ vs. $0-21 \%$, respectively ${ }^{26-27}$.

Some studies suggest that response to neoadjuvant chemotherapy, as evaluated by radiological or biochemical tests, may be a selection criterion for synchronous or metachronous resection ${ }^{28-29}$. For patients with complete or partial response to chemotherapy, subsequent metachronous resection resulted in a median OS of 36 months, compared to 11 months in patients who received chemotherapy alone ${ }^{28}$.

FOLFIRNOX or gemcitabine plus nab-paclitaxel are established as the standard systematic chemotherapy and are suggested for liver metastases. FOLFIRNOX is composed of fluorouracil, leucovorin, irinotecan, and oxaliplatin. This regimen significantly prolonged the median OS compared to gemcitabine monotherapy (11.1 months vs. 6.7 months). However, this treatment regimen was associated with severe toxicity. Another treatment alternative, with a relatively better safety profile, is the combination of gemcitabine and nab-paclitaxel, which achieved a median OS of 8.7 months ${ }^{26}$.

\section{Gastrointestinal stromal tumor (GIST)}

About 25\% of the GIST patients are diagnosed with synchronous distant metastases. The liver is one of the most influenced organs ${ }^{30}$. Treatment with tyrosine kinase inhibitors (TKIs) is the basic standard therapy for GIST liver metastases. Treatment with imatinib (a TKI), followed by resection, has been suggested for the treatment of GIST liver metastases ${ }^{31}$, which has greatly improved the outcomes of GIST patients. Xia et al. also reported that surgery plus imatinib significantly improved the overall survival of patients compared with imatinib monotherapy ${ }^{32}$. The response rate achieved with imatinib was $80 \%$, leading to an improvement in median overall survival from less than 2 years to 5 years $^{33}$.

The NCCN guidelines recommend that surgical resection can be performed under the following circumstances: limited metastatic disease, resistance to TKI drugs, and favorable response to chemotherapy and TKI drugs by previously unresectable lesions. While, Studies have shown that the 1-, 3- and 5-year survival rate after liver resection ranges from 93\%-100\%, 67.9\%-89.5\%, and 50\%-76\%, respectively. The median overall survival reported was 41.8 months. The 1-, 3- and 5-year DFS have been reported in the following ranges: $63.4 \%-87.5 \%, 26.1 \%-42 \%$, and 10\%-14\%, respectively ${ }^{34-41}$.

Age $>55$ years is a predictor of worse outcomes. R0 resection has been proven to dramatically improve the prognosis of GIST liver metastases, compared with R1/ $\mathrm{R} 2$ resection. Additionally, preoperative administration of imatinib is beneficial to achieve prolonged $0^{31-41}$.

\section{Kidney}

About $30 \%$ of the patients with renal cell carcinoma (RCC) develop extra-renal metastasis, and liver involvement is seen in $20.3 \%$ of the patients. Except for CRC and NETs, renal cancer metastases represent $6 \%-8 \%$ of the remaining secondary liver malignancies ${ }^{42}$. Liver metastases are definitely associated with a worse prognosis. Among them, only about $25 \%$ of metastatic lesions are solitary ${ }^{43}$. Additionally, liver metastases are always accompanied by extra-hepatic metastasis. Only about $1 \%$ of patients with RCC liver metastases received liver resection ${ }^{44}$.

A recent study reviewed liver resection for RCC liver metastases. A total of 76\%-100\% patients received R0 resection. The median survival after surgery was 15-142 months. The median DFS ranged from 7.2-27 months. The $1-, 3-$, and 5-year OS rates varied from 69\%-100\%, 26\%$83.3 \%$, and $0 \%-62 \%$, respectively ${ }^{42}$.

\section{Breast cancer}

Approximately, $20-30 \%$ of the patients with breast cancer develop distant metastasis. Bone is the most frequently influenced organ with an involvement rate of $85 \%$, followed by the liver ( $40 \%-50 \%)$. However, only about $5 \%$ of the metastases are restricted to the liver, leading to a very limited resection ratio for breast cancer liver metastases (BCLM) ${ }^{45}$. 
A retrospective study concluded that compared to drug therapy alone, BCLM resection can significantly improve the 3-year overall survival rate (81\% vs. $51 \%)$. Furthermore, a combination of surgery and medical strategy is also essential for long-term survival of patients with BCLM. Preoperative chemotherapy is considered crucial for the improvement of surgical outcomes. Adam et al. reported that the 5-year survival rates in patients with partial response, stable disease, and progressive disease were $42 \%, 12 \%$, and $0 \%$, respectively 5 . Results from M.D. Anderson Center showed that estrogen receptor positive $(\mathrm{ER}+)$ or progesterone receptor positive $(\mathrm{PR}+) \mathrm{BCLM}$ patients may have better overall survival after surgery (ER+: 76.8 months vs 23.1 months; PR+: 61.8 months vs 31.9 months $)^{46}$.

To achieve the best results, Golse et al. set a strict criteria for the selection of patients for resection, which included: metastases lesion $<4-5 \mathrm{~cm}$, single or not requiring major hepatectomy, $\mathrm{R} 0$ resection, stable disease after neoadjuvant systemic therapy, and a delay between primary and secondary lesions longer than 1 or 2 years ${ }^{45}$.

Unresectable CRLM can be converted into resectable tumors after chemotherapy. However, considering that BCLM resectability is based on the response to neoadjuvant systemic therapy, no study has been reported in which unresectable BCLM can be converted into resectable BCLM $^{45}$.

The human epidermal growth factor receptor 2-positive (HER2+) represents approximately 15\% of breast cancer patients. Introduction of anti-HER2 therapy has dramatically improved the overall survival of these patients. The 5-year survival rate of metastatic breast cancer patients was $37 \%{ }^{47}$. In contrast, the median overall survival was only 20 months for HER2-negative patients ${ }^{48}$.

\section{Conclusions}

For each subtype of malignancy, treatment strategy and prognosis vary significantly due to the differences in biological characteristics. NETs and GIST liver metastases have been well studied and the criteria used for these malignancies may be used as a guideline for other cancers. Kidney and breast cancer are relatively moderate and tend to have favorable prognoses. Surgical decisions should be made based on systematic treatment. Treatment options for pancreatic cancer are limited, with no major breakthrough achieved in decades. Despite the fact that some progress has been made with respect to synchronous resection, surgical resection for metastases should be critically evaluated. We did not review immunotherapy for each subtype of cancer liver metastases because immunotherapy is based on gene characteristics rather than histological origins, which may lead to a new era in the treatment of metastasis.

\section{Conflict of interest}

The authors declare that there are no conflicts of interest.

\section{Acknowledgements}

There is no financial sponsor for this review.

\section{References}

1. Vilchez VR. Gedaly, Liver transplantation for the treatment of neuroendocrine liver metastases, Best practice \& research. Clinical endocrinology \& metabolism. (016; 30: 141-147.

2. Saxena A, Chua TC, Perera M, et al Surgical resection of hepatic metastases from neuroendocrine neoplasms: a systematic review, Surg Oncol. 2012; 21: e131-141.

3. Gomez D, Malik HZ, Al-Mukthar A, et al. Hepatic resection for metastatic gastrointestinal and pancreatic neuroendocrine tumours: outcome and prognostic predictors. HPB : the official journal of the International Hepato Pancreato Biliary Association. 2007; 9: 345-351.

4. Nigri G, Petrucciani N, Debs T, et al. Treatment options for PNET liver metastases: a systematic review. World journal of surgical oncology. 2018; 16: 142.

5. Elias D, Lasser P, Ducreux M, et al. Liver resection (and associated extrahepatic resections) for metastatic well-differentiated endocrine tumors: A 15-year single center prospective study. Surgery. 133: 375382.

6. Sarmiento JM, Que FG, Grant CS, et al. Concurrent resections of pancreatic islet cell cancers with synchronous hepatic metastases: outcomes of an aggressive approach. Surgery. 2002; 132: 976-982; discussion 982-973.

7. Ronald, Chamberlain, David, et al. Hepatic neuroendocrine metastases: does intervention alter outcomes?

8. Chen H, Hardacre JM, Uzar A, et al. Isolated liver metastases from neuroendocrine tumors: does resection prolong survival?. 1998; 187 : 88.

9. Nave H, Mössinger E, Feist $H$, et al. Surgery as primary treatment in patients with liver metastases from carcinoid tumors: A retrospective, unicentric study over 13 years. Surgery. 2001; 129: 170-175.

10. Jaeck D, Oussoultzoglou E, Bachellier P, et al. Hepatic metastases of gastroenteropancreatic neuroendocrine tumors: safe hepatic surgery. World journal of surgery. 2001; 25: 689-692.

11. Cozzi PJ, Englund R, Morris DL. Cryotherapy treatment of patients with hepatic metastases from neuroendocrine tumors. Cancer. 1995; 76: 501-509.

12. Grozinsky-Glasberg S, Kaltsas G, Kaltsatou M, et al. Hepatic intraarterial therapies in metastatic neuroendocrine tumors: lessons from clinical practice. Endocrine. 2018; 60: 499-509.

13. Mazzaferro V, Pulvirenti A, Coppa J. Neuroendocrine tumors metastatic to the liver: how to select patients for liver transplantation?. Journal of hepatology. 2007; 47: 460-466.

14. Alagusundaramoorthy SS, Gedaly R. Role of surgery and transplantation in the treatment of hepatic metastases from neuroendocrine tumor. World journal of gastroenterology. 2014; 20: 14348-14358.

15. Valle JW, Eatock M, Clueit B, et al. A systematic review of non-surgical treatments for pancreatic neuroendocrine tumours, Cancer treatment reviews. 2014; 40: 376-389.

16. Rinke A, Müller HH, Schade-Brittinger C, et al. Placebo-controlled, double-blind, prospective, randomized study on the effect of 
octreotide LAR in the control of tumor growth in patients with metastatic neuroendocrine midgut tumors: a report from the PROMID Study Group. Journal of clinical oncology : official journal of the American Society of Clinical Oncology. 2009; 27: 4656-4663.

17. Caplin ME, Pavel M, Cwikla JB, et al. Lanreotide in metastatic enteropancreatic neuroendocrine tumors. The New England journal of medicine. 2014; 371: 224-233.

18. Jann H, Denecke T, Koch $M$, et al. Impact of octreotide long-acting release on tumour growth control as a first-line treatment in neuroendocrine tumours of pancreatic origin. Neuroendocrinology. 2013; 98: 137-143.

19. Wolin EM. The expanding role of somatostatin analogs in the management of neuroendocrine tumors. 2012; 5: 161-168.

20. Strosberg JR, Fine RL, Choi J. First-line chemotherapy with capecitabine and temozolomide in patients with metastatic pancreatic endocrine carcinomas. Cancer. 2011; 117: 268-275.

21. Yamaguchi T, Machida N, Morizane C, et al. Multicenter retrospective analysis of systemic chemotherapy for advanced neuroendocrine carcinoma of the digestive system. Cancer Science 2014; 105: 1176-1181.

22. Frilling A, Modlin IM, Kidd M, et al. Recommendations for management of patients with neuroendocrine liver metastases. The Lancet Oncology. 2014; 15: e8-e21.

23. Page AJ, Weiss MJ, Pawlik TM. Surgical management of noncolorectal cancer liver metastases. Cancer. 2014; 120: 3111-3121.

24. Gleisner AL, Assumpcao L, Cameron JL, et al. Is resection of periampullary or pancreatic adenocarcinoma with synchronous hepatic metastasis justified?. Cancer. 2007; 110; 2484-2492.

25. Bellon E, Gebauer F, Tachezy M, et al. Pancreatic cancer and liver metastases: state of the art. Updates in surgery. 2016; 68: 247-251.

26. Lee RC, Kanhere H, Trochsler M, et al. Pancreatic, periampullary and biliary cancer with liver metastases: Should we consider resection in selected cases?. World journal of gastrointestinal oncology. 2018; 10 : 211-220.

27. Tachezy M, Gebauer F, Janot M, et al. Synchronous resections of hepatic oligometastatic pancreatic cancer: Disputing a principle in a time of safe pancreatic operations in a retrospective multicenter analysis. Surgery. 2016; 160: 136-144.

28. Crippa S, Bittoni A, Sebastiani E, et al. Is there a role for surgical resection in patients with pancreatic cancer with liver metastases responding to chemotherapy?. European journal of surgical oncology : the journal of the European Society of Surgical Oncology and the British Association of Surgical Oncology. 2016; 42: 1533-1539.

29. Hackert T, Niesen W, Hinz U, et al. Radical surgery of oligometastatic pancreatic cancer. European journal of surgical oncology : the journal of the European Society of Surgical Oncology and the British Association of Surgical Oncology. 2017; 43: 358-363.

30. Miettinen M, Lasota J. Gastrointestinal stromal tumors. Gastroenterology clinics of North America. 2013; 42: 399-415.

31. Machairas N, Prodromidou A, Molmenti E, et al. Management of liver metastases from gastrointestinal stromal tumors: where do we stand? Journal of gastrointestinal oncology. 2017; 8: 1100-1108.

32. Xia L, Zhang MM, Ji L, et al. Resection combined with imatinib therapy for liver metastases of gastrointestinal stromal tumors. Surgery today. 2010; 40: 936-942.

33. Blanke CD, Rankin C, Demetri GD, et al Phase III randomized, intergroup trial assessing imatinib mesylate at two dose levels in patients with unresectable or metastatic gastrointestinal stromal tumors expressing the kit receptor tyrosine kinase: S0033. Journal of clinical oncology : official journal of the American Society of Clinical Oncology. 2008; 26: 626-632.

34. Seesing MF, Tielen R, van Hillegersberg R, et al. Liver Surgery Working, Resection of liver metastases in patients with gastrointestinal stromal tumors in the imatinib era: A nationwide retrospective study. European journal of surgical oncology: the journal of the European Society of Surgical Oncology and the British Association of Surgical Oncology. 2016; 42: 1407-1413.

35. Brudvik KW, Patel SH, Roland CL, et al. Survival After Resection of Gastrointestinal Stromal Tumor and Sarcoma Liver Metastases in 146 Patients. Journal of gastrointestinal surgery : official journal of the Society for Surgery of the Alimentary Tract. 2015; 19: 1476-1483.

36. Morris AD, Maithel SK, Kooby DA. Management of Liver Metastases of Gastrointestinal Stromal Tumors. Springer International Publishing. 2017.

37. Cheung TT, Chok KS, Chan AC, et al. Analysis of long-term survival after hepatectomy for isolated liver metastasis of gastrointestinal stromal tumour. ANZ journal of surgery. 2014; 84: 827-831.

38. Turley RS, Peng PD, Reddy SK, et al. Hepatic resection for metastatic gastrointestinal stromal tumors in the tyrosine kinase inhibitor era. Cancer. 2012; 118: 3571-3578.

39. de la Fuente SG, Deneve JL, Parsons CM, et al. A comparison between patients with gastrointestinal stromal tumours diagnosed with isolated liver metastases and liver metastases plus sarcomatosis. HPB : the official journal of the International Hepato Pancreato Biliary Association. 2013; 15: 655-660.

40. Shima Y, Horimi T, Ishikawa T, et al. Aggressive surgery for liver metastases from gastrointestinal stromal tumors. Journal of hepatobiliary-pancreatic surgery. 2003; 10: 77-80.

41. Cananzi FC, Belgaumkar AP, Lorenzi B, et al. Liver surgery in the multidisciplinary management of gastrointestinal stromal tumour. ANZ journal of surgery. 2014; 84: E1-E8.

42. Pinotti E, Montuori M, Giani A, et al. Surgical treatment of liver metastases from kidney cancer: a systematic review. ANZ journal of surgery. 2019; 89: 32-37.

43. Ruys AT, Tanis PJ, Nagtegaal ID, et al. Surgical treatment of renal cell cancer liver metastases: a population-based study. Annals of surgical oncology. 2011; 18: 1932-1938.

44. Janzen NK, Kim HL, Figlin RA, et al. Surveillance after radical or partial nephrectomy for localized renal cell carcinoma and management of recurrent disease. Urologic Clinics of North America. 2003; 30: 843852.

45. Golse N, Adam R. Liver Metastases From Breast Cancer: What Role for Surgery? Indications and Results. Clinical breast cancer. 2017; 17: 256-265.

46. Nosher JL, Ahmed I, Patel AN, et al. Non-operative therapies for colorectal liver metastases. Journal of gastrointestinal oncology. 2015; 6: 224-240.

47. Rossi M, Carioli G, Bonifazi M, et al. Trastuzumab for HER2+ metastatic breast cancer in clinical practice: Cardiotoxicity and overall survival. European journal of cancer. 2016; 52: 41-49.

48. Llombart-Cussac A, Pivot X, Biganzoli L, et al. A prognostic factor index for overall survival in patients receiving first-line chemotherapy for HER2-negative advanced breast cancer: an analysis of the ATHENA trial. Breast. 2014; 23: 656-662. 